\title{
ANP Supermatrices: Lessons from Hierarchies
}

\author{
Orrin Cooper ${ }^{\mathrm{a}}$ and William C. Wedley ${ }^{\mathrm{b}}$ \\ ${ }^{a}$ Fogelman College of Business \& Economics, The University of Memphis, Memphis, TN, USA \\ ${ }^{b}$ Beedie School of Business, Simon Fraser University Burnaby, B. C., Canada
}

\begin{abstract}
The AHP can be referred to as a specific subset of decision models within the more general ANP. But ANP models are more complicated and perceived as difficult to understand. Viewing each column in an ANP supermatrix as a hierarchy provides clarity about how the priority vectors, clusters, and columns are combined in a supermatrix. Paying greater attention to the units of measurement and applying an AHP perspective provides answers to important questions that can significantly impact the quality of ANP decisions.
\end{abstract}

Keywords: Aggregation, Transformation, Unit of Measurement, Hierarchies, ANP

\section{Introduction}

The ability to take ratio pairwise comparisons, two elements at a time, and then transform them into priority values that become meaningful in a multicriteria decision model is one of the main appeals of the Analytic Hierarchy Process (AHP) and the Analytic Network Process (ANP). Although ANP can be thought as a more general approach to solve both hierarchy and network problems, AHP is still the most common method that is used. Reasons for this may be familiarity with the AHP, the ability to integrate it with other methods, or a reluctance to use the ANP due to its perceived complexity. To reduce the complexity and cognitive load, we utilize principles from AHP to analyze ANP procedures. This approach is helpful because (1) users are familiar with the AHP and (2) various essential ANP assumptions can be emphasized.

A key component of ANP complexity is the supermatrix, whether from a single network or from a more complex decision that contains several networks and hence multiple supermatrices. In either case, understanding the supermatrix is essential. We find it useful to consider each column of a supermatrix as a hierarchy and the aggregation of those hierarchal columns as the solution to the network problem. This perspective provides insight into the meaning of each of the elements in both the unweighted and weighted supermatrices. Then, we can focus on other important questions like: What are the units of measurement in each cluster, hierarchy, and network? How should vectors within the hierarchies/columns be combined? How can the hierarchies then be connected together so that we can calculate the relative influence of the alternatives and criteria within the entire network? How do we synthesize a network of hierarchies? Beginning with connecting clusters within a hierarchy, we can move to connecting the hierarchies within the entire network, and through many methods including matrix multiplication meaningful final priorities can be obtained.

\section{Literature Review}

The ANP is widely known and has been used to make decisions across many disciplines. The details of its development, the steps to build a model, and the mathematical proofs behind the method are well documented (Saaty, 2005). Some potential advantages of using the ANP are: to develop models in the way that we naturally think about problems; to capture dependencies among the elements in a decision; and to model complex decisions like in Benefits, Opportunities, Costs, and Risks (BOCR) models. One complaint is that an ANP model generally requires more pairwise comparisons than an AHP model. However, an ANP 
model can capture dependency that an AHP model cannot and methods exist to reduce the number of comparisons which can also improve the accuracy of the decision.

Pairwise comparisons are entered into a pairwise ratio matrix (PRM), ratio priorities are derived, and the resulting ratio vectors are entered into an unweighted Supermatrix (Saaty, 2005). The vectors in the unweighted supermatrix are then transformed to get a weighted supermatrix from which a network solution is determined. Throughout all these processes of derivation, weighting and aggregation, very little concern is given to what happens to units of measure. In part, this is because "... we do not need explicit knowledge of the underlying unit of measure to derive a ratio scale, yet the derived scale has a unit" (Wedley and Choo, 2011). As well, scales that are normalized to sum to unity have no particular object such as the ideal that is specified as the unit of measure. For these reasons, opaqueness, not transparency, exists for units of measure.

Understanding the unit of measurement in the different representations and transformations is crucial, especially so that they are not misunderstood and misused. For example, Choo et al. (1999) studied criteria weights and discovered that there is no consensus on the meaning or manner of deriving them. When those criteria weights are used to transform vectors or clusters of vectors, ratio values are changed to a new unit of measure. If new units in the weighted supermatrix do not capture dependencies in the ANP model, incorrect rankings can occur (Cooper and Liu, 2017). From a hierarchical perspective, Wedley and Choo (2011) illustrated that the unit of local priorities is an abstract object possessing the totality of vector values; and that the unit of global priorities is an object possessing the totality of all global values in the hierarchy. It should be noted that the process of changing units from local to global priorities in AHP is identical to columnar transformations in ANP when going from the unweighted to the weighted supermatrix. In essence, each column of the supermatrix is hierarchy in a network structure.

\section{Hypotheses/Objectives}

Using the existing literature about the unit of measurement and our proposed AHP perspective to view the supermatrix as columns of hierarchies will provide insight and clarity about the following items in an ANP supermatrix:

- How is a supermatrix organized?

- What do the elements in the supermatrix represent?

- Why is knowing the unit of measurement in each cluster and hierarchy of the unweighted and weighted supermatrix important?

- Why aren't the 1's in this column equal to the 1's in that column?

- How can cluster comparisons be adapted to capture the dependency in an ANP model?

- Are there other ways besides matrix multiplication to find the weights of the elements within the entire network?

\section{Research Design/Methodology}

Two examples are presented of a practical decision between 3 alternatives which are evaluated with respect to 3 clusters of criteria and which also has a known answer for comparison purposes. A general network with the relationships for the first element of the supermatrix is presented in Figure 1. While the it may look like three different networks we will show how via lessons from a hierarchy that these three networks are identical. One numerical example will serve to show how each column of a supermatrix can be viewed as a hierarchy and the other provides a contrasting example as to why the insights from this perspective can be helpful. Within columns of the unweighted supermatrix are one or more vectors analogous to the local weights of a hierarchy that sum to unity. As each column is viewed as a hierarchy and is added and integrated together we build both the unweighted and weighted Supermatrices. While weighted supermatrix values are global to columns, they are not global to the network. Likewise, while each column sums to 
unity, each column and its unit is generally not equal in importance. To get the overall importance for the entire network, differential values must be determined for the columns of the weighted supermatrix.
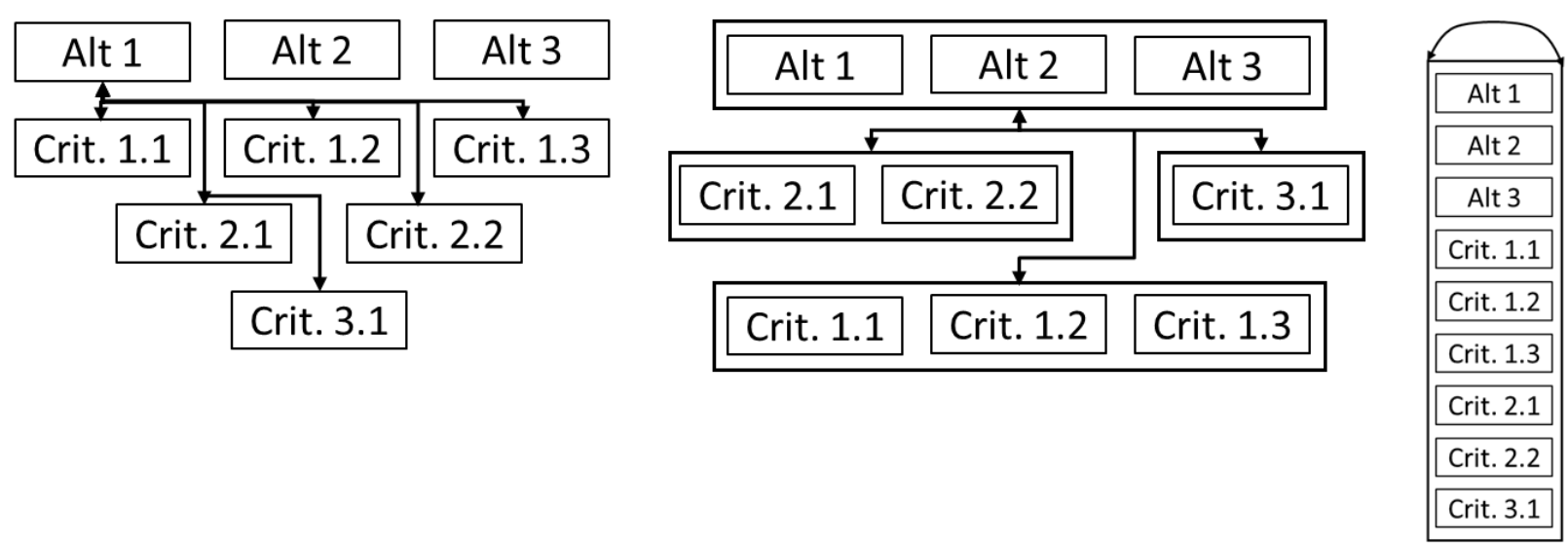

Figure 1 Three Identical Network Diagrams of the Hierarchy in the first element in the supermatrix

This can be done in many ways including linking and matrix multiplication. Acknowledging these concepts and using this step by step approach provides answers to the questions listed in the previous section. As each question is discussed we update the Supermatrices accordingly and can compare the differences between the two examples to provide the needed motivation to apply the proposed framework in other ANP decisions.

\section{Limitations}

We recognize this as a shift in the focus and perspective that can provide clarity about important steps in making an ANP decision. Likewise, it opens other questions such as how to integrate the direction of the influence in an ANP network. We look forward to valuable feedback about the proposed framework and its implications.

\section{Conclusions}

While hierarchies and networks may have seemed rather unrelated, the proposed perspective brings them together in a way that provides clarity and insight as to why units of measurement are important throughout the entire decision-making process. The relationships between each column in the supermatrix become clear. This framework highlights why decision makers need to pay particular attention and be deliberate as they aggregate ratio vectors within the hierarchies and the hierarchies within the network. The need to capture the dependency between the priorities and the specified network is clarified through the examples. Viewing each column of an ANP supermatrix as a hierarchy provides relatable answers to important questions about ANP models in general. We hope this framework and the answers it provides will help more decision makers take advantage of some of the potential benefits of using the ANP and provide a direction and context for future research. 


\section{Key References}

Choo, E. U., Schoner, B., \& Wedley, W. C. (1999). Interpretation of criteria weigh in multicriteria decision making. Computers \& Industrial Engineering, 37 (3), 527-541.

Cooper, O., \& Liu, G. (2017). Achieving the desired level of dependency in ANP decision models. International Journal of the Analytic Hierarchy Process, 9(1).

Saaty, T. L. (2005). Theory and applications of the analytic network process: decision making with benefits, opportunities, costs, and risks. RWS publications.

Wedley, W. C., \& Choo, E. U. (2011). Multi-criteria ratios: What is the unit? Journal of Multi-Criteria Decision Analysis, 18 (3-4), 161-171. 